\title{
Diacronie
}

Studi di Storia Contemporanea

$N^{\circ} 14,2 \mid 2013$

Processo penale, politica, opinione pubblica (secoli XVIII-XX)

\section{Prefazione n. 14 - agosto 2013}

\section{Jacopo Bassi e Deborah Paci}

\section{(2) OpenEdition \\ Journals}

\section{Edizione digitale}

URL: http://journals.openedition.org/diacronie/121

DOI: $10.4000 /$ diacronie. 121

ISSN: 2038-0925

Editore

Association culturelle Diacronie

Notizia bibliografica digitale

Jacopo Bassi e Deborah Paci, « Prefazione n. 14 - agosto 2013 », Diacronie [Online], N 14, 2| 2013, Messo online il 01 août 2013, consultato il 22 septembre 2020. URL : http://journals.openedition.org/ diacronie/121; DOI : https://doi.org/10.4000/diacronie.121

Questo documento è stato generato automaticamente il 22 settembre 2020.

Creative Commons License 


\title{
Prefazione n. 14 - agosto 2013
}

\author{
Jacopo Bassi e Deborah Paci
}

1 Il numero 14 di Diacronie si compone di due distinte sezioni: il Laboratorio e un approfondimento, composto di tre saggi, sul tema della vena espansionista italiana.

2 Il Laboratorio è stato concepito come uno spazio di pubblicazione per i lavori svolti dagli studenti dei corsi di Laurea Magistrale; l'intento, quando l'idea è

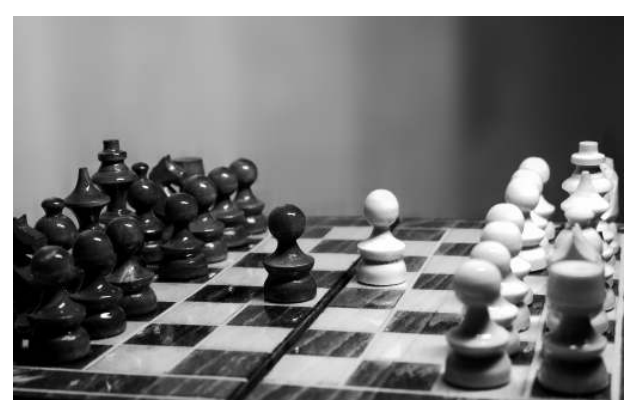
sorta, a partire da uno spunto della

Professoressa Francesca Sofia, era quello di dare visibilità agli argomenti e agli elaborati sviluppati nell'ambito delle lezioni dei corsi di Laurea. Dove sono presenti laboratori di ricerca a tutti gli effetti (pensiamo ad esempio ai casi francesi e brasiliano), in grado di coinvolgere anche gli studenti dei master, l'operazione è stata realizzata ed ha portato buoni risultati. Alle indubbie finalità didattiche (lo sviluppo della capacità di trattare un argomento affrontandolo attraverso diversi casi studio, ma partendo da premesse comuni; l'utilizzo della "cassetta degli attrezzi" dello storico; l'esercizio della scrittura; l'adattamento del proprio lavoro in vista di una pubblicazione), che competono però più agli interessi del versante accademico, si unisce la possibilità, per Diacronie e per i suoi lettori, di vedere in opera le prime fasi di costruzione di un saggio di storia. Per via della genesi degli elaborati e per come il lavoro degli studenti del corso di Storia delle istituzioni sociali e politiche europee si è sviluppato, invitiamo i lettori a considerare il laboratorio come un unicum, i cui saggi, pur nelle loro specificità, portano avanti una comune riflessione.

3 Per parte nostra la speranza è quella di avere contribuito al livellamento dello scalino che ancor oggi permane fra i luoghi di formazione dello storico (i corsi di laurea e il master) e gli spazi dedicati alla diffusione della ricerca.

4 La sezione Un posto al sole raccoglie invece tre saggi dedicati ai disegni operati dai governi italiani per affermare il paese sulla scena internazionale, seppur in diversi ambiti d'azione, cercando di affrancarsi dal ruolo di Potenza minore. 
L'aspirazione espansionistica dell'Italia nel continente africano ha una lunga storia che affonda le sue radici nella seconda metà dell'ottocento e che con l'avvento del fascismo raggiunse il suo apice.

Il saggio di Michele Pandolfo, La Somalia coloniale: una storia ai margini della memoria italiana, ripercorre le tappe dell'interesse da parte italiana nei confronti della Somalia, dall'Ottocento sino al termine della Seconda guerra mondiale, quando le Nazioni Unite affidarono all'Italia uno speciale protettorato sul paese: l'Amministrazione fiduciaria italiana sulla Somalia (Afis).

7 La conquista dell'Etiopia e la proclamazione dell'Impero rappresentarono il momento di massimo consenso degli italiani nei confronti del regime fascista. Agli occhi della comunità internazionale il fascismo pretese di indossare le vesti dell'erede designato della missione universale e imperiale che era stata di Roma antica. João Fábio Bertonha con Paranoie fasciste? Il volontariato in favore dell'Etiopia durante la guerra del 1935-1936 affronta un aspetto della guerra in Etiopia trattato marginalmente dalla storiografia italiana: quello dei timori da parte della diplomazia italiana rispetto all'eventualità che potesse sorgere non soltanto una resistenza etiope all'invasione italiana, ma anche svilupparsi una forma di volontariato antifascista internazionale.

Nel corso degli anni Venti, e con maggiore intensità negli anni Trenta, si andò diffondendo l'idea secondo la quale l'Italia avesse acquisito, dopo secoli di oscurità, il diritto di alzare la voce sulla scena internazionale. Il saggio di Rubén Domínguez Méndez, El fascismo italiano y la Exposición Internacional de Barcelona de 1929, tratta della partecipazione dell'Italia all'Esposizione Internazionale di Barcellona del 1929: un'occasione per il fascismo italiano di affermare di fronte alla comunità internazionale i traguardi conseguiti dal regime nei primi anni della sua attività di governo, nonché la solidità dei rapporti con la dittatura di Primo de Rivera. Nella prospettiva fascista la credibilità e l'autorevolezza in ambito internazionale avrebbe contribuito a modificare l'immagine degli italiani all'estero: abituati a emigrare in ogni parte del mondo alla ricerca di un lavoro, non si sarebbero visti obbligati a chinare il capo di fronte a nuovi padroni. Il fascismo voleva infondere a tutti gli italiani, sia residenti nel Regno, sia nelle comunità sparse nel mondo, la cognizione di appartenere ad una grande nazione che poteva sedere al tavolo delle trattative con le altre nazioni e ricercare il suo "posto al sole".

Chiude il numero la consueta sezione dedicata alle recensioni.

Buona lettura.

\section{AUTORI}

\section{JACOPO BASSI}

Nel 2006 consegue la Laurea Triennale in « Storia del mondo contemporaneo » presso l'Università di Bologna sostenendo una tesi in Storia e istituzioni della Chiesa ortodossa dal titolo Tra Costantinopoli e Atene: Il passaggio delle diocesi dell'Epiro all'amministrazione della Chiesa di Grecia e la 
'Praxis' del 1928, relatore il Professor Enrico Morini. Nel 2007, nel quadro del programma di scambio Erasmus, ha frequentato per un trimestre l'École Normale Supérieure (ENS) di Parigi; ha effettuato un periodo di soggiorno durante i mesi di gennaio e febbraio 2008 presso l'École Française d' Athènes, sotto il tutorato del dottor Anastassios Anastassiadis, membro del comitato scientifico di questa istituzione. Nel luglio 2008 ha discusso la Tesi Specialistica in Storia della Chiesa - relatore il Professor Umberto Mazzone, correlatore il Professor Enrico Morini - dal titolo Epiro crocifisso o liberato? La Chiesa ortodossa in Epiro e in Albania meridionale nel XX secolo (1912-1967). Ha lavorato come redattore per la casa editrice L'Inventairee ha curato il progetto Dictionnaire universel des femmes créatrices - secteur "Femmes du livre" in corso di pubblicazione presso Editions des Femmes. Attualmente collabora con la casa editrice Il Mulino alla creazione dell'archivio digitale dei libri del Mulino, Darwinbooks e con la casa editrice Carocci.

\section{DEBORAH PACI}

Nel 2006 consegue la Laurea Triennale in «Storia contemporanea» presso l'Università di Bologna discutendo una tesi sulla battaglia autonomista condotta dal Partito d'Azione in Valle d'Aosta e in Sicilia durante il triennio 1943-1946, dal titolo Il contributo del Partito d'Azione nella lotta per le autonomie. Sicilia e Valle d'Aosta a confronto (1943-1946). Nel 2007 partecipa al programma internazionale «Cursus intégré franco-italien d' histoire européenne comparée», promosso dall'Università di Bologna e dall'Université Paris VII - Denis Diderot. Nel 2008 consegue un doppio titolo di Laurea Specialistica in «Storia d'Europa» e di Master 2 «Histoire et civilisations comparées», sostenendo una tesi sulla ricezione del pensiero di Pierre-Joseph Proudhon presso i fuorusciti italiani in Francia dal titolo Dall'anarchia al federalismo. La tradizione proudhoniana nei Fuorusciti italiani in Francia. L'attenzione in questo studio è stata rivolta alla lettura delle teorie proudhoniane operata dal sociologo Georges Gurvitch e alla sua trasmissione negli ambienti del fuoruscitismo italiano negli anni tra le due guerre. Tra il 2009 e il 2011 è stata borsista presso l'Ecole Française de Rome e visiting student presso l'University of Malta. Nel 2013 consegue un dottorato di ricerca in cotutela in «Scienze storiche» presso l'Università di Padova e in «Histoire» presso Université de Nice Sophia-Antipolis, svolgendo una ricerca sul mito del Risorgimento mediterraneo e sul progetto imperialista fascista in Corsica e a Malta negli anni tra le due guerre. Fa parte del Centro Interuniversitario di Storia Culturale (CSC) e del Centre de la Méditerranée Moderne et Contemporaine (CMMC). Attualmente Assegnista di ricerca presso l'Università Ca' Foscari di Venezia sta conducendo uno studio sulle politiche identitarie e sull'immaginario insulare nelle isole del Mediterraneo e del Baltico. 